Article

\title{
Assessment of Arundo donax Fibers for Oil Spill Recovery Applications
}

\author{
Vincenzo Fiore $^{1, *(\mathbb{D}}$, Elpida Piperopoulos ${ }^{2}$ (I) and Luigi Calabrese ${ }^{2}(\mathbb{D})$ \\ 1 Department of Engineering, University of Palermo, Viale delle Scienze, Edificio 6, 90128 Palermo, Italy \\ 2 Department of Engineering, University of Messina, Contrada di Dio, Sant'Agata, 98166 Messina, Italy \\ * Correspondence: vincenzo.fiore@unipa.it
}

Received: 19 July 2019; Accepted: 16 August 2019; Published: 21 August 2019

\begin{abstract}
In the last years, natural fibers are increasingly investigated as an oil recovery system in order to overcome the oil spillage phenomena, thus preserving environment and aquatic life. In particular, lignocellulose-based fibers have recently been employed with promising results. In such a context, the aim of this paper is to assess the oil sorption capability of natural fibers extracted from the stem of the giant reed Arundo donax L., a perennial rhizomatous grass belonging to the Poaceae family that grows naturally all around the world thanks to its ability to tolerate different climatic conditions. Sorption tests in several pollutants and water as a reference were carried out. The fibers have absorption capacities that are about five to six times their weight. Depending on the high absorption kinetics, possible applicative interests can be identified. Eventually, depending on the fiber size, adsorption properties were related to the microstructure and morphology of Arundo donax fibers.
\end{abstract}

Keywords: natural fibers; Arundo donax; oil recovery; sorption capacity; morphology

\section{Introduction}

Oil represents one of the most important raw material sources for the production of synthetic polymers and chemicals worldwide. Consequently, there is a high risk of oil spills whenever oil is explored, transported, and stored or its derivatives are used, thus leading to significant environmental impact [1] and consequently causing an adverse impact on economic, social, and ecological systems. Due to this, several efforts aimed at oil spill recovery were done in the last years to identify the optimal cleaning-up technologies by means of physical, chemical, and biological methods [2]. Among these techniques, the physical approach through the application of sorbent materials is considered one of the most efficient and high-performing methods [3]. In this context, the main challenge is to find cheap, eco-friendly, reusable, and recyclable materials that possess a high adsorbent capacity in order to simplify disposal procedures at the end of its life and optimize the costs to benefits ratio. It is worth noting that, up to now, synthetic polymers, mineral materials, and natural biomass-based oil sorbents have been considered as oil sorbent materials. Despite synthetic polymers, such as polypropylene [4], polyurethane [5] and methacrylate [6] are considered ideal materials for oil spillage clean-up due to their low density, good hydrophobicity, optimal physical and chemical resistance, and great efficiency in the oil removal from oil-water mixtures. They are made from non-biodegradable and expensive oil by-products. Mineral materials, such as vermiculite, exfoliated graphite, and fly ash, can be also used even if most of them are characterized by poor buoyancy and low oil sorption capacity [7]. In order to find new eco-friendly materials that are useful for this application, more attention has been focused in the last decades on biomass-based oil sorbents, such as lignocellulosic fibers or particles.

In particular, the use as sorbent materials for a crude oil of mixed leave residues, mixed sawdust, sisal and coir fibers, sponge-gourd, and silk-floss was evaluated by Annunciado et al. [8], who showed that silk-floss evidenced the best oil sorption capacity. Adhithya et al. [9] evidenced the great 
efficiency of bamboo fibers for the removal of both vegetable and synthetic oils from oil-water mixtures. Yang et al. [7] focused their attention on the use of platanus fruit fibers for the preparation of efficient oil sorbents. In particular, they evaluated the effect of chemical modification with acetic anhydride of the above fruit fibers, showing that this treatment greatly enhances their sorption capacity for various oils and organic solvents.

Liu et al. [10] covered with polyethylene wax and alkyl ketene dimer a pulverized wastepaper with the aim of increasing its hydrophobicity. The experimental campaign evidenced that a dramatic increase of the adsorbing capacity of the raw material can be obtained even if the modified materials cannot be considered renewable and biodegradable. Key attributes for good absorbent materials are represented by the combination and optimization properties, such as the pollutant absorption capacity, high selectivity to water or reusability, and preservation of economic and environmental characteristics (e.g., costs, green sustainability, and easy recyclability).

A cost effective, ultralight, elastic, and highly recyclable material with enhanced absorption capability was manufactured by treating renewable cellulose fibers through a simple and eco-friendly microfibrillation method and freeze-drying steps [11]. In addition to the high adsorption capacity, the resulting material showed an excellent flexibility and elasticity, i.e., it can be reused for at least 30 adsorbing/squeezing cycles without any reduction of its oil sorption capability.

Moreover, biomass carbon aerogels with excellent oil sorption ability were produced from sisal leaves by means of alkalization, bleaching, freeze-drying, and carbonization processes [12] whereas porous carbon spheres with a high rate of adsorption kinetics (i.e., sorption saturation can be achieved within 1 to $2 \mathrm{~min}$ ) were derived through a simple carbonization of the fruit of Liquidambar formosana [13]. Further investigations were focalized on the evaluation of the oil absorption capacity of raw barley straw [14], kapok fiber [15], and raw bagasse [16].

In such a context, the present paper aims to assess the oil sorption properties of lignocellulosic fibers extracted from the stem of the giant reed Arundo donax L., a perennial rhizomatous grass belonging to the Poaceae family that grows naturally all around the world thanks to its ability to tolerate different climatic conditions. A relevant requirement that strongly stimulate its application in this context is the intrinsic porous microstructure that potentially integrates well with this application. Likewise, the use of Arundo donax (AD) could represent a cost-effective and non-toxic solution to an oil spill issue, with beneficial effects for the environment.

This invasive and aggressive plant is able to grow 4 to $10 \mathrm{~cm}$ per day under optimal conditions (i.e., cultivation) [17] and its deep and permanent roots play a key role in the prevention of soil erosion in addition to performing their primary task of ensuring efficient water absorption [18].

With regards to its range of applications, Arundo donax stem is used for the production of musical woodwind instruments $[19,20]$, fences, trellises, stakes for plants, windbreaks, sun shelters [21], as a fiber source for printing paper [22], and as a source of biomass for chemical feedstocks and for energy production. In particular, Papazoglou et al. [23] evaluated the growth of Arundo donax L. on soil irrigated with cadmium and nickel solutions to evaluate the impact of these heavy metals on its growth and photosynthesis. The experimental results showed that this plant can be cultivated in contaminated soils, thus providing biomass for energy production purposes. Moreover, Flores et al. [24] showed that this non-wood plant can also be used for the manufacturing of chipboard panels suitable for indoor use, since they reached the minimum structural parameters for that use (i.e., acceptable resistance compared to the wood panels available on the market). Fiore et al. [25] extracted fibers from the outer part of the stem, showing that Arundo donax fibers represent a valid alternative to other common natural fibers as reinforcement in polymer-based composites. After this preliminary work, the use of Arundo donax fibers in epoxy resin [26] and poly (lactic acid) [27] was assessed. Moreover, the beneficial effect of plasma treatment on the adhesion between fibers extracted from the leaves of Arundo donax $\mathrm{L}$. plant and a bio-based epoxy matrix was demonstrated [28]. Concerning cementitious-based materials, the partial replacement of sand in concrete mixes using giant reed ash and air-dried giant reed fibers 
was evaluated by Ismail and Jaeel [29]. The experimental results highlighted that noticeable increments of the compressive strength can be achieved through the above replacement.

The considerable industrial flexibility of this material suggests its potential use in contexts where the chemical and physical characteristics of the fibers are integrated to their mechanical properties. For this reason, although it is not yet investigated, their use as oil spillage systems represents a stimulating and innovative challenge in the panorama of the current literature. This study aimed to evaluate the effectiveness of its application in this context, evaluating its potential use for different classes of liquid pollutants.

\section{Materials and Methods}

\subsection{Preparation of Arundo Donax Fibers}

The natural fibers used for oil sorption characterization were acquired from the stem of the giant reed Arundo donax L. and were collected after flowering in a local plantation area of Palermo (Italy). After the fresh plant collection, stems with outer and inner average diameters equal to 25 and $17 \mathrm{~mm}$ were selected and accurately treated in order to separate the stem from the foliage. Afterwards, the stems were cut into small parts and according to ASABE S358.3 (2012), dried at $103^{\circ} \mathrm{C}$ for $24 \mathrm{~h}$ in an oven to remove all the moisture content. Fibers with lengths in the range 100-160 mm were extracted from the stem by mechanical separation. In particular, the outer part of the culm was manually decorticated with the help of blades to obtain thin strips from which fibers were easily separated with the aid of a scalpel and a Leica optical microscope model MS5 (Leica Microsystems, Wetzlar, Germany). Finally, the obtained long fibers of Arundo donax were chopped at different lengths in order to evaluate the effect of fiber size on the sorption capabilities for different oil pollutants. In particular, three batches were prepared at varying lengths of Arundo donax fiber in the range of 300-2500 $\mu \mathrm{m}$, coding them AF-L, AF-M, and AF-S for large, medium, and small fiber sizes, respectively. Details are reported in Table 1. Fibers' size morphology, and geometry were evaluated by environmental scanning electron microscopy (ESEM, Quanta 450, FEI, Hillsboro, OR, USA).

Table 1. Arundo donax fiber sample details.

\begin{tabular}{cccc}
\hline Code & Notes & Length $[\mu \mathrm{m}]$ & Diameter $[\mu \mathrm{m}]$ \\
\hline AF-S & Small AD Fibers & $300-1000$ & $100-300$ \\
AF-M & Medium AD Fibers & $1000-1500$ & $300-500$ \\
AF-L & Large AD Fibers & $1500-2500$ & $500-800$ \\
\hline
\end{tabular}

\subsection{Sorption Capacity Experiment}

In order to carry out absorption tests on AD fiber and avoid their dispersion in the pollutants, the fiber batches were placed inside a plastic bag. Sorption tests were performed by equilibrating AD fiber sample bags in $250 \mathrm{~mL}$ of different commercial oils open to air at room temperature and under slow stirring $(200 \mathrm{rpm})$. In particular, four commercial oils were used for the sorption experiments: Kerosene, virgin naphtha, pump oil, and crude oil. For comparison, sorption of distilled water was also performed. Some characteristics of the selected oils are summarized in Table 2 (where $\rho$ and $\mu$ are the density and dynamic viscosity of the pollutants, respectively).

Table 2. Density $(\rho)$ and dynamic viscosity $(\mu)$ of selected oils.

\begin{tabular}{cccccc}
\hline & Water & $\begin{array}{c}\text { Virgin } \\
\text { Naphtha }\end{array}$ & Kerosene & Crude Oil & Pump Oil \\
\hline$\rho\left(\mathrm{kg} / \mathrm{m}^{3}\right)$ & 1000 & 630 & 780 & 890 & 858 \\
$\mu(\mathrm{Pa} \mathrm{s})$ & 0.0010 & 0.0012 & 0.0019 & 0.2710 & 0.1231 \\
\hline
\end{tabular}


At an increasing immersion time in the pollutant liquid, the Arundo donax bags were accurately removed from the beaker. The impregnated samples were places in a watch glass, open to air for $30 \mathrm{~s}$ in order to release the residual pollutant and subsequently weighed. Furthermore, sorption measurements on empty bags were performed as a reference and used to extrapolate the sorption properties of Arundo donax fibers. The mass adsorption capacity was calculated using the following equation:

$$
Q_{t}=\frac{m_{t}-m_{0}}{m_{0}} \times 100,
$$

where $Q_{t}$ is the sorption capacity (in percentage) of the fiber at sorption time $t$, and $m_{0}$ and $m_{t}$ are the initial weight (at instant $t=0 \mathrm{~s}$ ) and the weight after sorption time $t$ of the AD sample (reduced by the bag contribute), respectively. The sorption measurements were performed until sample weight stabilization was reached. This value was identified as the sorption capacity at saturation.

\section{Results and Discussion}

\subsection{Morphology of Arundo Donax Fibers}

In Figure 1, the surface morphology of an Arundo donax fiber is shown. As other lignocellulosic fibers, the cross-section morphology of Arundo donax fibers is constituted by several fibrils (known also as fiber cells) along the fiber direction that are linked to each other by pectin and other non-cellulosic compounds $[25,30]$, which act as a bonding matrix, thus forming a mechanically effective fiber bundle.

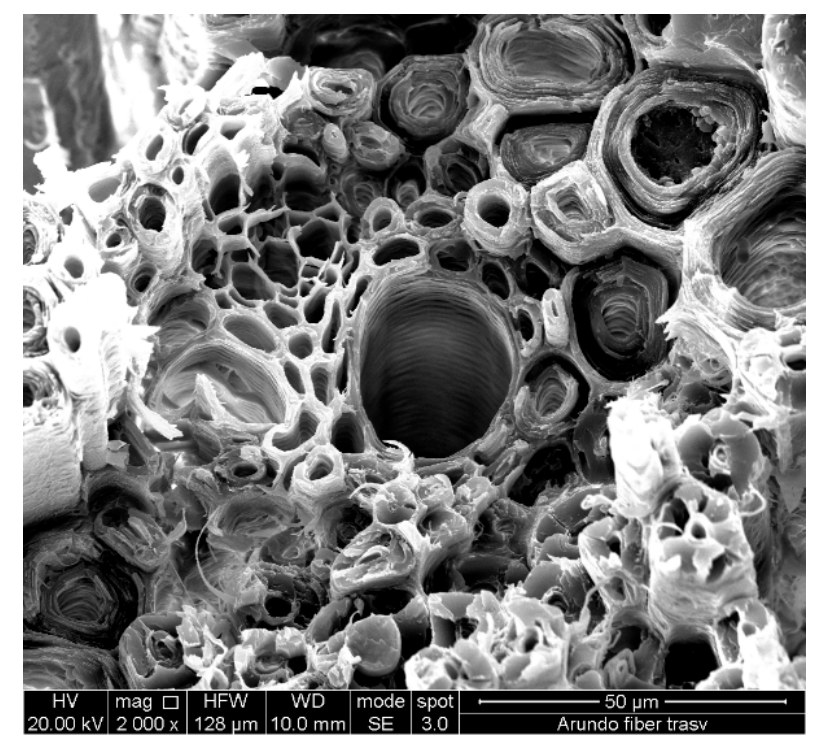

Figure 1. SEM micrograph of cross section of Arundo fiber.

A morphological structure with vascular bundles can be clearly identified. The fiber cells are constituted by a non-regular-sized polygonal shape with a central hole, named the lumen [25], that gives the bundle its lightweight morphology. In particular, by analyzing the micrograph reported in Figure 1, it is possible to detail the morphology of the fiber cells' structure. Arundo donax fiber has large porous lumens with very wide and dispersed channels with a circular-type geometry along the preferential orientation of the fibrils. However, the channel size is not regular, with a wide dispersion in diameter. Furthermore, defects and heterogeneities can be evidenced on the structure. The high diameter size variation of fiber cells and lumens have a relevant effect on the physic-chemical and mechanical performances of the AD fibers [30]. Concerning the application of Arundo donax fibers as an oil spill remedy, a key point that needs to be addressed is therefore to assess the structure morphology and to relate it with the AD fiber performances to be able to effectively define the relationship between the 
structure and sorption performances of the AD fibers used as sorbent particulate. In such a context, the morphological peculiarity of being lightweight and the high internal porosity observed in the AD fibers plays a crucial role in the adsorption performances of the sorbent at varying oil pollutant present as environmental contaminants.

Arundo donax fibers show a porous orthotropic morphology with lamellar structure that identifies a preferential orientation and several interconnected pores, as observed in Figure 1. Consequently, depending on the particulate size used as absorbent material, more or less regular morphological structures can be obtained. On this concern, samples belonging to three different sizes were analyzed. The AF-L (Figure 2a) has a mean length and width size higher than $1500 \mu \mathrm{m}$ and $500 \mu \mathrm{m}$, respectively. The morphology is characterized by circular channels of the fiber cells, with fibers regularly oriented along the fiber length. Some cell interconnections can be identified among the fibrils, obtaining a porous beehive-like inter-communicating structure. AF-M samples (Figure $2 b$ ) are characterized by a mean length size is in the range of 1000 to $1500 \mu \mathrm{m}$. It is constituted by smaller Arundo donax particles than the AF-L one. The cellular structure of the fibers, although a decreased channel size and amount is observed, is still preserved. However, considering the reduced width $(\sim 300-500 \mu \mathrm{m}) \mathrm{a}$ limited fiber cells interconnection occurred. In Figure 2c, a micrgraphy of the AF-S sample is reported. The particulate sorbent is characterized by a mean length and width size lower than 1000 and $300 \mu \mathrm{m}$, respectively. The original beehive structure, clearly observed in AF-L samples, is completely destroyed. The lightweight structure of Arundo donax fibers with large porous channels (subsequently to their low density) is drastically modified. Preferential oriented fibrils along the fiber length are still present, but the interconnected porous structure, due to the shredding step, was damaged, thus limiting its potential efficiency as a sorbate container.

\subsection{Sorption Performances}

The sorption capacity evolution, $Q_{t}(\%)$, indicated as the pollutant uptake, at varying sorption time for all AD sorbent fibers for different oil pollutants is shown in Figure 3.

All curves evidence a monotone trend with a progressive increase at increasing adsorption time until a constant weight at saturation is reached. In particular, three steps in the sorption evolution can be identified:

I. At first, at low sorption time (range 0-30 s), a rapid increase of the sorption capacity, due to a high sorption rate, can be highlighted.

II. Afterwards, at an intermediate sorption time, the pollutant uptake increases with a reduced sorption rate as confirmed by the low uptake versus time slope. This region occurs at different time ranges depending on the selected pollutant (rough range of about 30 to $60 \mathrm{~s}$ ).

III. Finally, the pollutant uptake reaches a plateau, due to a stabilization of the sorption capacity, where an equilibrium condition occurs. In this stage, only a very limited increase in the sorption capacity takes place due to the reduced active sorbent sites available in the bulk and surface of the AD fibers for oil sorption and entrapment [31].

By analyzing AF-L samples, Figure 3a, high absorption performances at saturation were observed in the water and pump oil: $788 \%$ and $611 \%$, respectively. Conversely, the lowest sorption capabilities were observed in the crude oil pollutant with a sorbate uptake at a saturation of $443 \%$.

Progressively, at an increasing fiber size in the AD sorbent, the pollutant uptake appears to be apparently similar, although a slight deviation in trend can be found. For example, by analyzing the crude oil in the AF-L batch, the adsorption at saturation is the lowest than the other pollutants. Instead, kerosene and virgin nafta pollutant induced the lowest sorption capacity in AF-M and AF-S batches (in the range 460-470 for the AF-M batch and 425-435 for the AF-S batch. 


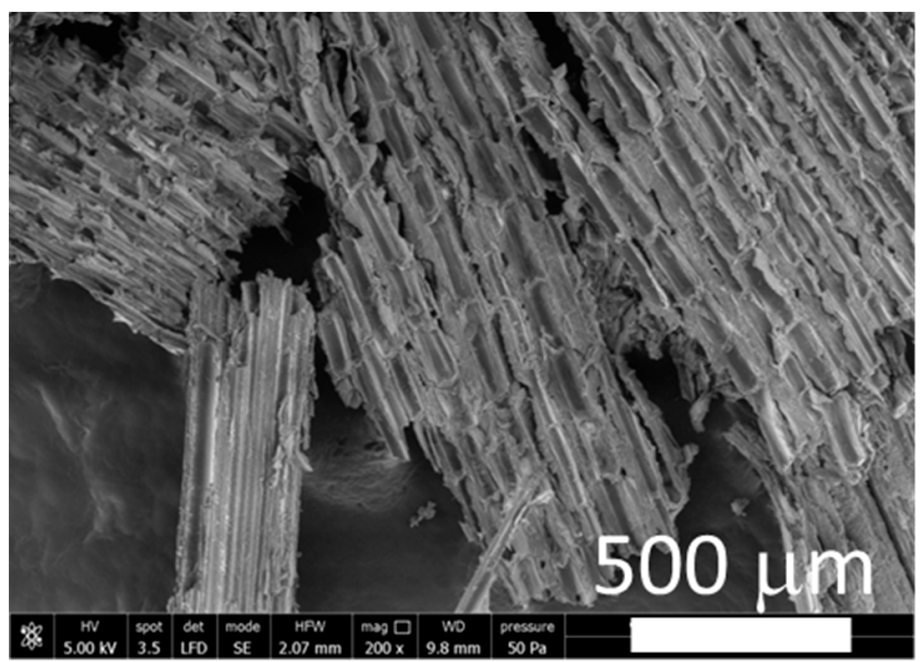

(a)

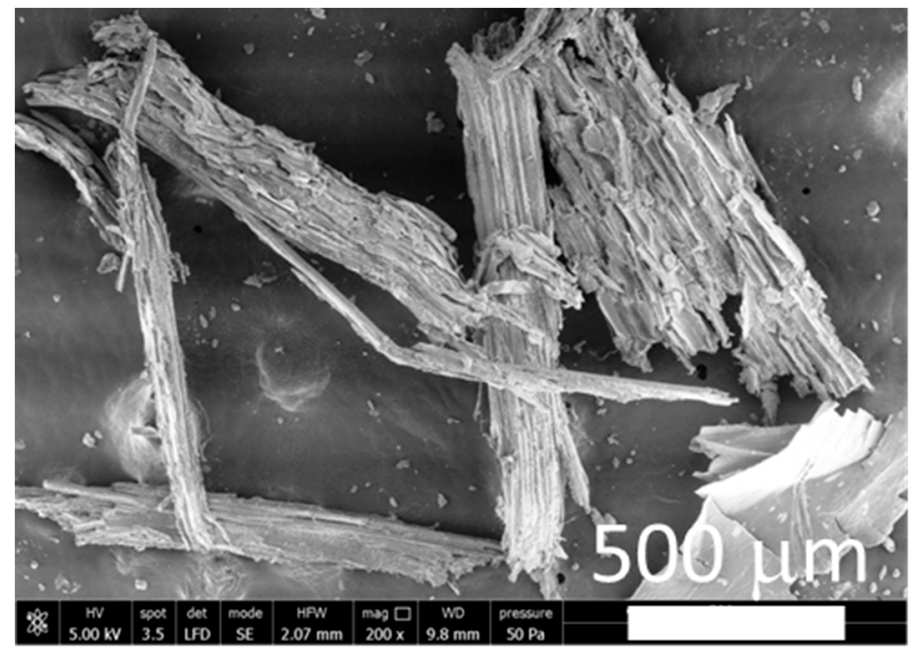

(b)

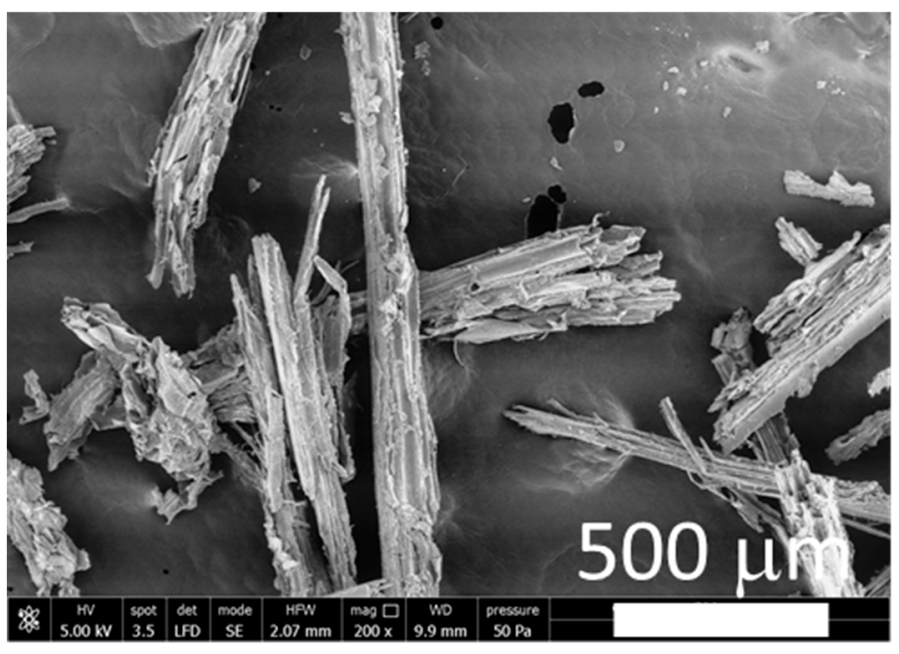

(c)

Figure 2. SEM images of (a) large-, (b) medium-, and (c) small-shaped Arundo donax fibers. 


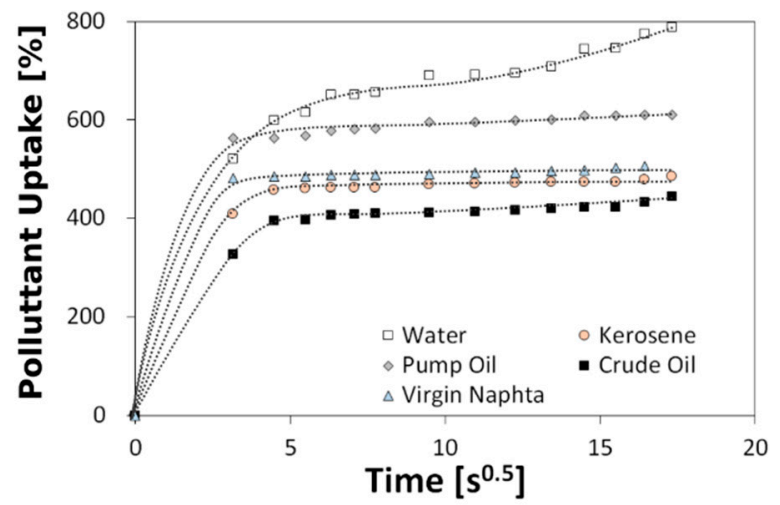

a)

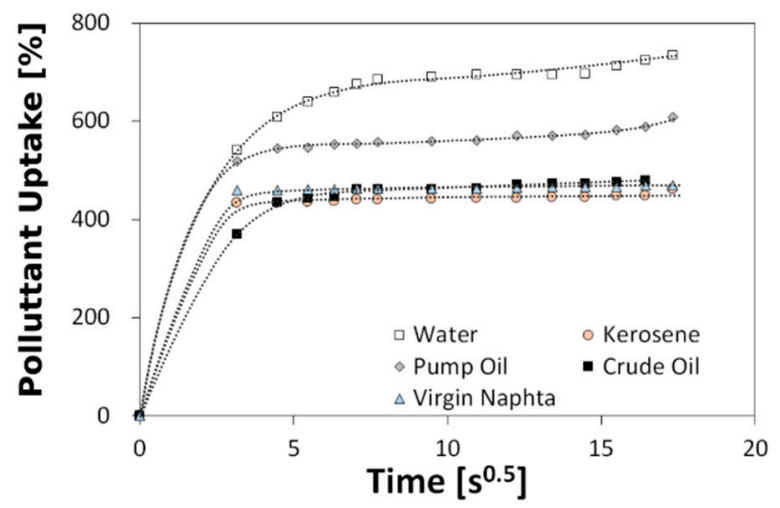

b)

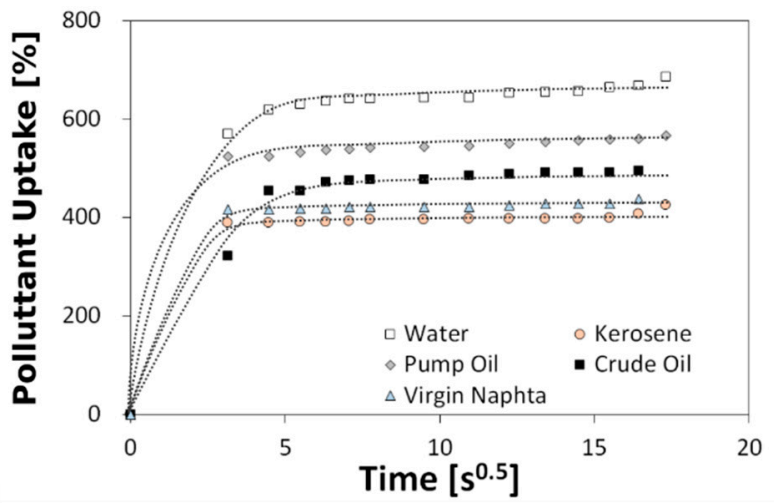

C)

Figure 3. Sorption capacity at increasing time for all pollutants of AF-L (a), AF-M (b), and AF-S (c) samples.

This implies that the size of the fibers leads to an interaction modification of the material surface and bulk with each specific pollutant, thus affecting its adsorbent capacities. In order to better assess the effect of the sorbent fiber size on absorption performances, Figure 4 shows the uptake trend at increasing time for two reference liquids, at low and high viscosity: Pump oil and water, in Figure 4a,b, respectively. The pump oil pollutant does not show a significant modification in the uptake trend at increasing particle size. Although, a difference in uptake at saturation can be evidenced. In particular, for an increasing AD fiber size, the pump oil uptake at saturation increases by about $10 \%$, from 567 to 610 for AF-S and AF-L, respectively (step 2 in Figure 4a). This discrepancy is also visible at a low sorption time, step 1 in Figure 4a, where the AF-L batch preserves a relevant higher pump oil uptake compared to the AF-S one. 


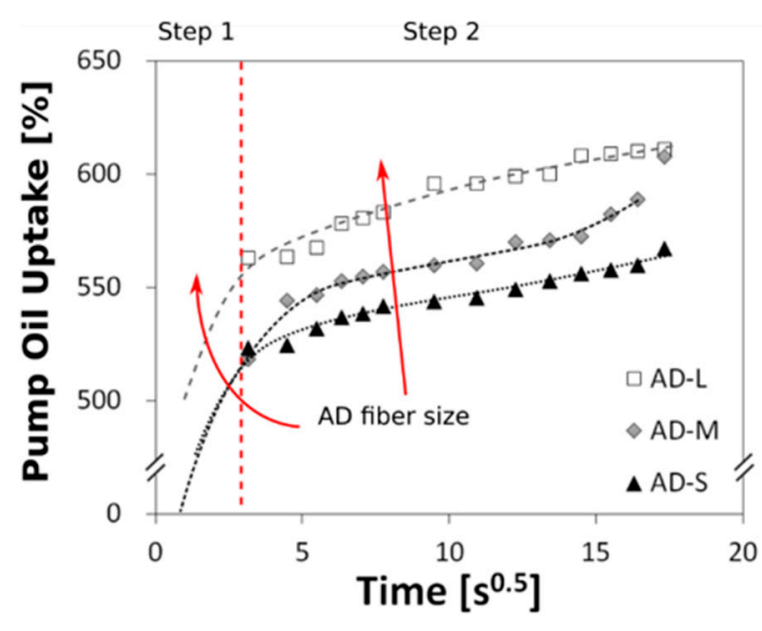

(a)

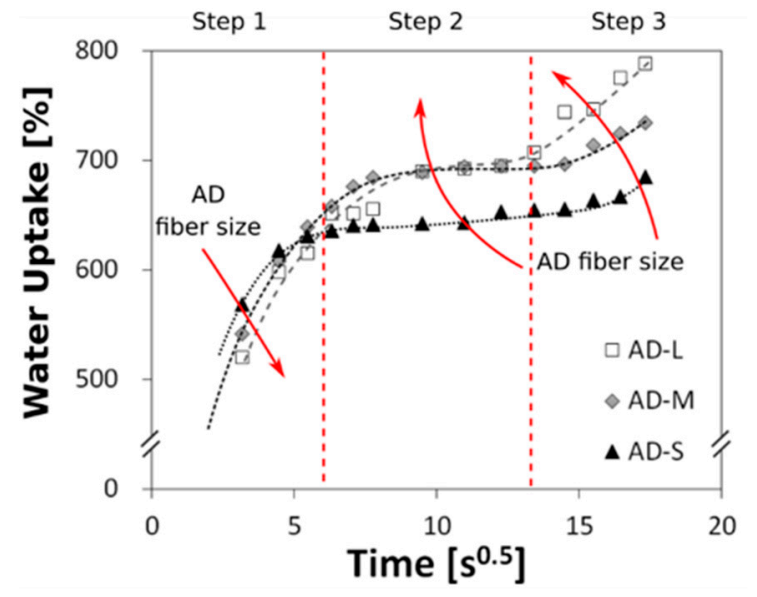

(b)

Figure 4. Sorption capacity at increasing sorption time in (a) water and (b) pump oil of AF-L, AF-M, and AF-S samples.

Different considerations can be extrapolated analyzing the sorption trend in water for Arundo donax fibers. It is worth noting that in water, several phenomena compete in the sorption process.

In particular, the initial phase (step 1 in Figure $4 b$ ) is slight kinetically favored in small-sized AD fibers. Conversely, the water uptake at saturation (step 3 in Figure $4 \mathrm{~b}$ ) is very high for the large-sized fibers. In fact, AF-M and mainly AF-L batches show a very extensive intermediate stage (step 2 in Figure 4 b), in which a slow but progressive increase in weight can be observed. This trend was not observed for AF-S specimens.

In summary, by analyzing the pump oil uptake trend at an increasing time, a bilinear trend can be identified. The initial phase at short times is associated with the rapid absorption phenomena that occurs thanks to a coupled action of mass transfer (i.e., transport phenomena from the pollutant solution toward the adsorbent surface) and intra-particle diffusion (i.e., diffusion phenomena toward the surface pores). The second phase can be identified when the uptake plateau occurs. In this stage, the absorption equilibrium is reached. This phase is identifiable by a quite horizontal straight line indicating the independence of the pollutant uptake percentage from time. In fact, as confirmed by Wahi et al. [32] in their study on sago bark fiber waste for the removal of oil from palm oil mill effluent (POME), a saturation step is reached when the adsorption-desorption process that occurs at saturated sorbent surfaces takes place. They ascribed the quite constant oil removal to the limited availability of sorbent surfaces for oil entrapment. Compatible consideration can be argued for AD fibers. It is worth noting that Arundo donax fibers, compared of sago bark fibers, are characterized by much faster adsorption kinetics and almost compatible adsorption values at saturation, assessing a more effective costs:benefits ratio.

Conversely, using water, a further intermediate step takes place. This step extends over a quite wide range (range: $\sim 50-250 \mathrm{~s}$ ). This stage can be related to the coupled action of two competing mechanisms: (i) The film diffusion, where the diffusion of the liquid film from the sorbent surface toward the fiber bulk occurs, and (ii) the surface interactions of the sorbent liquid on the active sites that gradually decreases, increasing the uptake.

Step 1 is relatively fast considering the rapid superficial absorption of the water and its diffusion on the superficial pores of the AD fibers. Instead, the intermediate stage (step 2 in Figure $4 \mathrm{~b}$ ) is related to the diffusion of the adsorbate in the structural interconnected channels of the porous adsorbent fibers [33].

The first phase, related to the pollutant interaction with the adsorbent, is influenced by the hydrophilic nature of AD fibers and is strongly favored for a large surface area. In such a context, 
the slope of the water uptake curve versus time is steeper as the smaller Arundo donax fiber size. Afterward, step 2, which is the intermediate stage, can be linked to the diffusion of adsorbate within the microporosity of the AD fiber structure. A catalytic contribution could be offered by the tortuosity of the fiber surface that acts as an active site for oil adsorption enhancing sorption efficiency [34]. As evidenced by the SEM images (Figure 2), these interconnections are evident in large-shaped fibers. In fact, AF-L samples are characterized by a well-structured morphology with ordered fibrils and circular-shaped channel longitudinal to the fibrils' direction. The large interconnection among these channels exalt the water sorption in this stage. Instead, the AF-S samples highlighted a very fine structure, comparable to the micro-fibrils' size. Therefore, a large amount of the porous fibrillar structure has been significantly damaged or destroyed.

These considerations indicate that the sorption mechanism is influenced by the fiber morphology and its porous microstructure if preserved, especially during the sorption of high viscose oils. Al-Din et al. [35] evidenced that the pores can make oil enter into the sorbent internal sections more easily, enhancing the sorption process and giving, in this way, a significant role to the sorption performance in oil spilling. Moreover, higher mean-sized samples (and consequently low density samples for the channels' presence) increase and speed up the sorption capabilities of pollutants. Therefore, step 2 for small fibers (AF-S) is short and the water uptake saturation is rapidly reached, with a lower maximum adsorption level than the AF-L batch for which the liquid has spread inside the porous structure, thus increasing the adsorption efficiency per unit of weight.

These considerations are summarized in Figure 5. The AF-L batch is characterized, as reported in SEM images in Figure 2, by a bamboo-like structure characterized by interconnected porous channels. These porous channels have a key role in the high sorption capacity of the material. In fact, adsorbed oil may fill them, enhancing the pollutant uptake. In the AF-M sample, the number of interconnection channels is limited, therefore the pollutant sorption is an obstacle toward the inner porous channels due to sub-optima mass transport. Therefore, the adsorption is mainly performed externally on the fibers' surface. This aspect is drastically exalted in the AF-S batch, where the interconnection channels are completely absent and only surface interaction with the pollutant oil can take place.

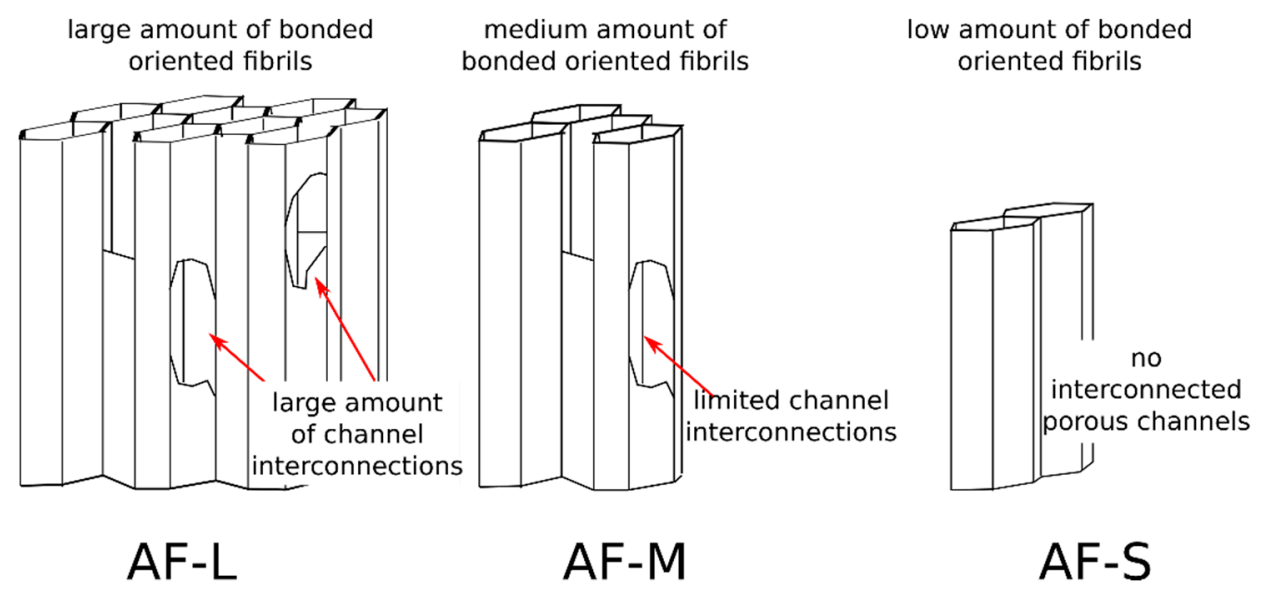

Figure 5. Schematic representation of samples' morphology.

In order to better compare the sorption capabilities' evolution at varying pollutant and sorbent size, Table 3 summarizes the equilibrium sorption capacity, $S e$, at equilibrium time, te, for all Arundo donax fiber batches at varying pollutants. It is worth noting that in water sorbate, all AD fibers are able to guarantee high sorption performances, with $S e$ values in the range of $788 \%$ to $684 \%$, with the highest value for the AF-L batch. Furthermore, a very high equilibrium time, te, can be highlighted. This indicates a good interaction of Arundo donax fibers with water, thanks to a surface hydrophilic behavior. At the same time, as previously discussed, the intra-particellar diffusion of low viscosity liquid takes place at a long sorption time. That extends the sorption stabilization at longer times in low 
viscosity sorbate liquids. Instead, with oil pollutants, the sorption capacity is lower than with water liquid coupled to a low equilibrium time, indicating that a fast sorption saturation condition is reached.

Table 3. Sorption capacity and time at equilibrium parameters for all liquids and all investigated AD fiber batches.

\begin{tabular}{|c|c|c|c|c|c|c|}
\hline & & Water & Kerosene & $\begin{array}{l}\text { Virgin } \\
\text { Naphtha }\end{array}$ & Pump Oil & Crude Oil \\
\hline \multirow{2}{*}{ AF-L } & $\mathrm{Se}(\%)$ & $788.0 \pm 39.4$ & $485.1 \pm 24.3$ & $505.8 \pm 25.3$ & $610.9 \pm 30.5$ & $443.4 \pm 22.2$ \\
\hline & te $(s)$ & $290.0 \pm 17.3$ & $33.3 \pm 5.8$ & $20.0 \pm 10$ & $80.0 \pm 17.3$ & $46.7 \pm 5.8$ \\
\hline \multirow{2}{*}{ AF-M } & $\mathrm{Se}(\%)$ & $734.3 \pm 36.7$ & $461.1 \pm 23.1$ & $469.7 \pm 23.5$ & $607.6 \pm 30.4$ & $484.4 \pm 24.2$ \\
\hline & te $(s)$ & $280.0 \pm 17.3$ & $23.3 \pm 5.8$ & $16.7 \pm 5.8$ & $53.3 \pm 5.8$ & $53.3 \pm 5.8$ \\
\hline \multirow[b]{2}{*}{ AF-S } & $\mathrm{Se}(\%)$ & $684.7 \pm 34.2$ & $425.3 \pm 21.3$ & $437.9 \pm 21.9$ & $567.2 \pm 28.4$ & $517.9 \pm 25.9$ \\
\hline & $t e(s)$ & $270.0 \pm 30.0$ & $16.7 \pm 5.8$ & $13.3 \pm 5.8$ & $43.3 \pm 5.8$ & $56.7 \pm 5.8$ \\
\hline
\end{tabular}

All samples showed an equilibrium time, $t e$, in the range 13-80 s, significantly lower than water (270-290 s). In particular, all the investigated samples showed a good affinity with pump oil, revealing among the analyzed oils, the highest sorption capacity. In particular, the AF-L sample reached $610.9 \%$ of the saturation sorption capacity, approaching the result obtained in water even if in less than a third of time. Conversely, AF-S evidences the highest equilibrium sorption value, among the other samples, in crude oil (517.9\%). This behavior could be related, probably to the high density and viscosity, $890 \mathrm{~kg}$ $\mathrm{m}^{-3}$ and $0.27 \mathrm{~Pa} \mathrm{~s}$, respectively [36], of the crude oil compared with the other pollutant oils. Due to the peculiar characteristics of the crude oil, its diffusion in interconnected channels is limited and the adsorption is, therefore, mainly ascribed to the superficial interaction of the fiber with the pollutant. Instead, in high viscosity oil, the pollutant sorption is favored on smaller sizes and fewer channel AD fibers (such as the AF-S batch) than those with more interconnected channels and a larger particulate size (such as the AF-M and AF-L batches).

Instead, for lighter and less viscous pollutants (i.e., kerosene and virgin naphtha oils), the liquid diffusion toward the interconnected channels is favored. Thus, it is confirmed by analyzing the performances of AF-L sample that shows a high equilibrium sorption capability in these liquids. The surface interaction of the fiber with the pollutant liquid have a relevant role on sorption performances. As indicated by Dong et al. [37], the wettability of the surface with the sorbate liquid determines specific sorption mechanisms. In particular, three conditions can be schemed: (i) A bad wettability and adhesiveness oil fiber can lead to an easy loss of oil drops from pores of fibrous filter with subsequent low sorption capabilities; (ii) for intermediate wettability and weak adhesiveness, oil droplets are attached to the fiber surface. Although, due to a sub-optimal adhesion, they can be easily detached, limiting the sorption capabilities; (iii) finally, if the fiber presents high wettability and excellent adhesion properties, oil droplets can be captured and adsorbed by the fiber. In these conditions, the higher sorption capabilities can be forecasted if the liquid-fiber surface interaction is the driving force in the sorption mechanism.

Further information can be acquired by evaluating the sorption performances of AD-sized fibers at varying pollutant viscosity. In this concern, a dimensionless parameter that relates the sorption performances with fiber size was defined (coded as ESS—effect of sorbent size) as in the following:

$$
\operatorname{ESS}_{i}[\%]=\frac{m_{i}-m_{L}}{m_{L}} \times 100,
$$

where $m_{L}$ is the sorption capacity (\%) of the AF-L AD fibers at a specific pollutant. $m_{i}$ is the sorption capacity (\%) in the same pollutant of AF-M or AF-S batches, obtaining the ESSM and ESSS parameter, respectively. A negative ESS value indicates that the sorption capacity of the large-sized AD fiber is higher than the smaller one. Therefore, with high or low values, this parameter indicates a relevant contribute of the fiber size on the adsorption performances of the AD fibers in the specific pollutant. 
Figure 6 compares the evolution of the ESSM and ESSS indices at an increasing pollutant viscosity. It is possible to evidence a monotonous trend, with a progressive growth of ESSM and ESSS values at increasing liquid viscosity. In particular, the index has a negative value in low viscosity pollutants. Afterwards, it progressively increases until a positive value is reached for liquids with a viscosity higher than about $0.2 \mathrm{~Pa}$ s. These results indicate that in low viscosity oil pollutants, the large-sized fibers have a higher sorption efficiency than the small-sized one. On the other hand, in high viscosity liquids, short $\mathrm{AD}$ fibers are more effective in sorption, showing higher adsorbent performances than long fibers. This behavior can be related to the Arundo donax fiber microstructure and the relative surface interaction with the liquid used as adsorbate. The presence of the structural porosity in the lumen and interconnected channels among the fibrils, as in the case of the AF-L batch, have a key role in the process. This morphology allows enhanced sorbent capabilities in low viscous liquids, where the mass flow into the porous fiber bulk is kinetically favored. Therefore, due to the low viscosity of the oil pollutant, a large amount of the internal channels can be imbibed, increasing the sorption efficiency of the natural fibers. In fact, the ESSS index is about $-15 \%$ compared to ESSM, which is approximately one-third lower (ESSM $\sim-5 \%$ ), confirming the larger effectiveness of large-sized fibers on pollutant uptake.

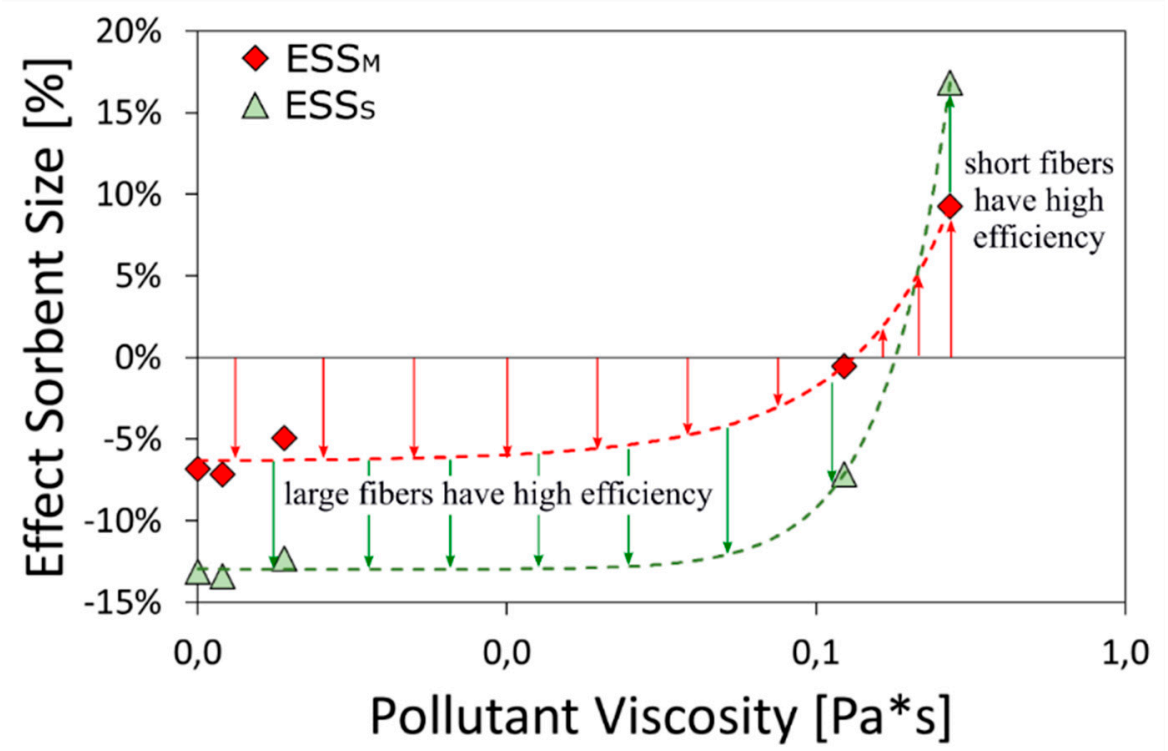

Figure 6. Evolution of the effect sorbent size index at varying pollutant viscosity.

On the other hand, for high pollutant viscosity, the mass diffusion toward the inner part of the fiber is significantly inhibited. The surface interaction can be considered as the main driving force in the sorption phenomenon. The AF-S batch, characterized by short-sized fiber with a subsequent large surface area, allows a higher sorption capacity than long-sized fiber batches. Considering that micro channel diffusion of pollutants is avoided in high viscous liquids, AF-L samples showed ineffective capabilities in these conditions. That occurs, as previously discussed, because of the low ability of large-sized fibers to host the adsorbed pollutant oil in their intrinsically interconnected porosity, limiting their sorption efficiency. In Figure 7, the high selectivity in the pump oil of AD fibers is shown. In Figure 7a, crude oil drops are poured into the water. Afterwards, the sorbent fibers, located in a plastic bag, are put into the contaminated beaker (Figure 7b). It is worth noting that after a few seconds, the sorbent material is dipped out and the crude oil drops clearly disappear (Figure 7c), remaining trapped in the adsorbent fibers (Figure 7d) and the water is cleaned up (Figure 7e). 


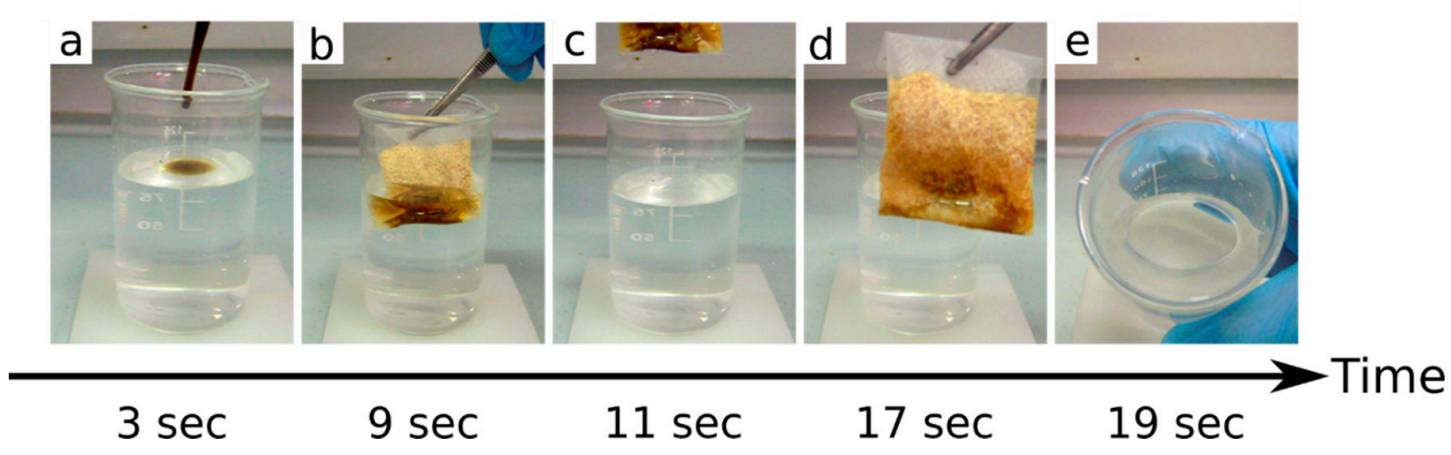

Figure 7. Oil adsorption steps in the water of Arundo donax fibers. (a) Crude oil drops are poured into water; (b) sorbent fibers are put into the beaker; (c) sorbent fibers are taken out and the crude oil drops disappear; (d) the oil remains trapped into the sorbent material; (e) the water is cleaned up.

This experimental evidence clarifies that although the AD fibers have a relevant adsorption capacity in water (Figure 3), the diffusion kinetics in oil pollutants is higher than in water, as identifiable by evaluating the shorter equilibrium time found in oil pollutants than in water solution (Table 3). Therefore, when fibers interact with a mixed oil-water solution, the fibers will be rapidly impregnated by oils, inhibiting kinetically the possible adsorption of water. As shown in Figure 7, Arundo donax fibers feasibly enabled the water to be decontaminated from crude oil in a few seconds. The results indicate a suitable adsorption efficiency (in terms of pollutant uptake speed) and oil selectivity. These two factors represent an effective and reliable precondition for the use of these natural fibers on the industrial scale for oil spill recovery, thanks also to the cheapness of the sorbent material, which can offset the sub-optimal adsorbing performance with respect to some new emerging materials [36,38,39]. According to [40], natural fibers are characterized by an acceptable sorption efficiency and capacity for different kinds of oil. Furthermore, these materials have been identified as promising fibers due to all advantages of agricultural wastes coupled to the high resistance, which exalt their stability and durability during its use.

\subsection{Morphological and Structural Aspects of Oil Spill AD Materials}

The morphology and microstructure of the natural fiber has a relevant role on the adsorption process of adsorbent materials. Surface asperities and local porosity are required to favor oil adsorption and trapping. The porosity size and distribution have key roles in the adsorption capacity of the sorbent material. Baker et al. [41] evidenced that the water selectivity of a membrane is related to the microstructure and chemistry of the material. Piperopoulos et al. [38], examining carbon nanotubes sponges for oil recovery applications, defined the sorption mechanism on porous structure in three main steps: (i) Oil molecules diffuse from bulk liquid and wet the sorbent; (ii) at the same time, physical (van der Waals forces between adsorbate molecules and the adsorbent surface) and/or chemical interactions take place, (iii) increasing the sorption time; liquid diffusion occurs with difficulty due to the limited hydrophilic or oleophilic (depending on the pollutant) active sites, not filling the internal micropores.

Analogously, Alaa El-Din et al. [35] ascribed the effective sorption performances of banana fibers to the irregular morphology and porous surface of the banana peels. The pores can promote the diffusion of oil toward the internal parts of the material more easily, thus favoring the sorption process.

On the other hand, Abdelwahab et al. [40] indicated that the oil adsorption capacity of palm fibers waste can be described as a single mechanism due to the monolayer coverage of the pollutant around the natural fiber, indicating that these fibers as excellent biomaterials for the removal of oil from aqueous environments.

The natural surface roughness of green adsorbent materials is an added value for this kind of application. Cui et al. [42] investigated adsorption phenomena on cattail fiber sorbents. They assessed 
that oil sorption evidenced by these natural fibers can be identified by combining different mechanisms, i.e., oil adsorption by interactions with waxes on the fiber surface and adsorption by physical trapping on the irregular fiber surface. Furthermore, the surface roughness and the open porous structure of the cattail fibers provide the high surface area able to guarantee suitable oil trapping.

Based on the previous remarks and on the supposed approaches, more competing adsorption stages could be recognized for Arundo donax sorption mechanism:

I Mass flow towards the fiber bulk (external mass transfer and film diffusion);

II Liquid diffusion toward the inner part (intraparticle diffusion).

For water adsorption, there is a further intermediate stage:

III Surface interaction on active sites and film diffusion.

Based on this scheme, evaluating the oil adsorption process in AF-L fibers, an intra-particle diffusion mechanism can be indicated as the rate limiting factor of the sorption process, and instead external mass transfer is generally rapid. So, in step 1, as represented in Figure 8, oil molecules diffuse from the bulk liquid and soak the AD sorbent. Subsequently, in step 2, oil diffusion into sorbent micropores takes place (Figure 8, step 2), increasing the oil sorption capacity of the AD fibers [42]. Instead, for the AF-S sample, the interconnection in the porous channels of the fibrils are almost absent. Therefore, the adsorption mechanism is carried out mainly by a single step (Figure 8, step 1) and saturation occurs more quickly (Table 3). In water, the first stage is fast, due to the superficial adsorption and the subsequent diffusion on the superficial pores. The intermediate stage is described by the water diffusion from the sorbent surface toward the fiber bulk and the surface interactions on the active sites.
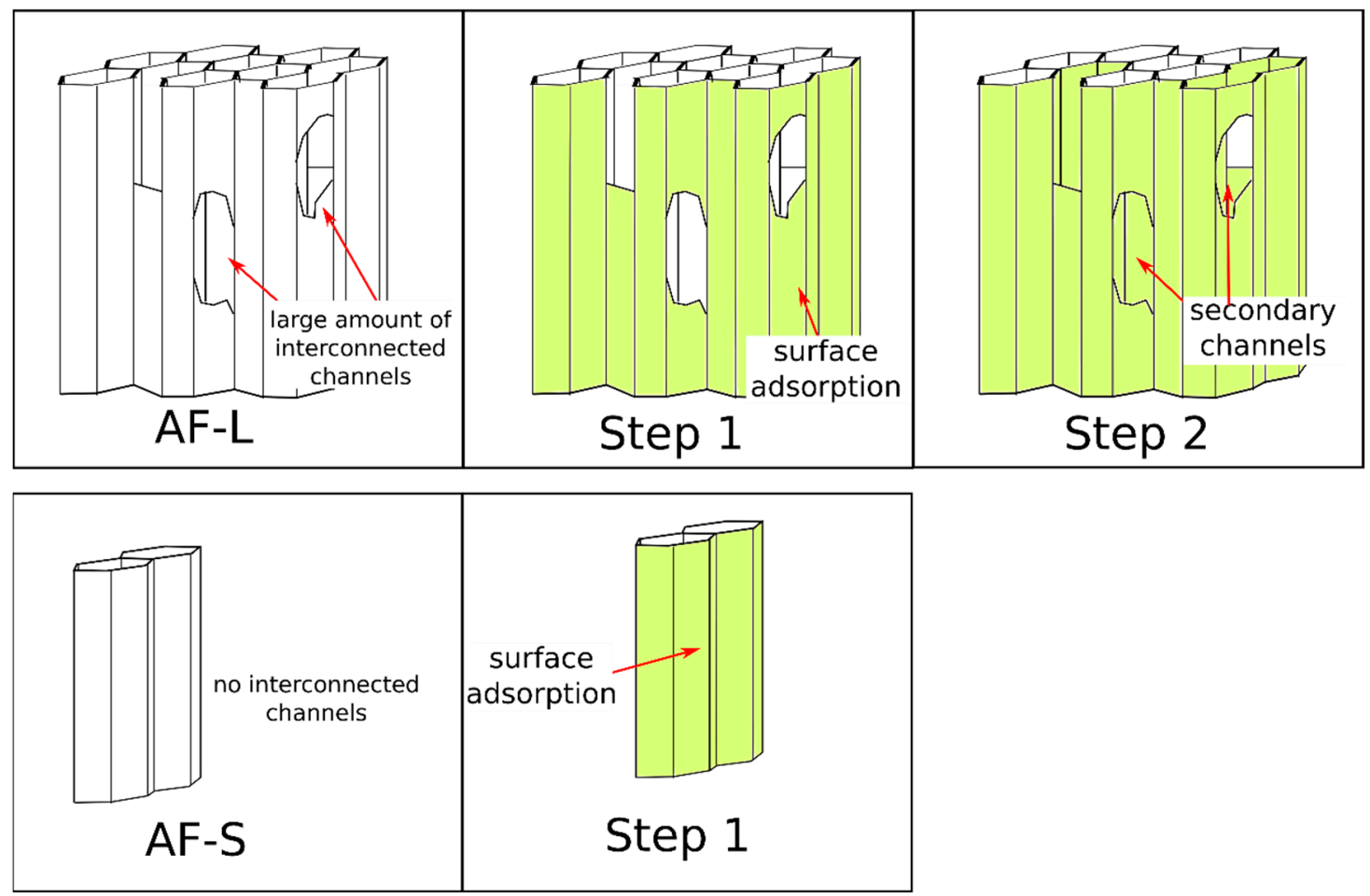

Figure 8. Sorption mechanism for AF-L and AF-S samples.

These preliminary results evidenced good potential applicability of this type of green adsorbents in oil recovery application, indicating a suitable interaction with large common oil pollutants. Certainly, further research developments are required in order to better relate the adsorption performances with 
the microstructure of the AD fiber. In addition, the assessment of the adsorption kinetic can represent a benefit in terms of an improved knowledge of the phenomenon to better discriminate the applicative value of these natural fibers.

In order to fill the gap compared to the other classes of materials currently in use for oil spill recovery, greater selectivity towards oils and absorption capacity are welcome, always preserving the environmental sustainability of the improved solutions. Therefore, the obtained results represent a stimulus aimed at enhancing this class of materials in unconventional application fields, such as oil spill recovery, allowing the use of a biodegradable, ecofriendly, and safe materials on this environmentallly sensitive application.

\section{Conclusions}

In this paper, the oil sorption capability of natural fibers extracted from the stem of the giant reed Arundo donax L. was assessed. Sorption tests were performed in several pollutants (e.g., kerosene, virgin naphtha, pump oil, and crude oil) and water as a reference. The fibers evidenced absorption capacities about five to six times their weight. Furthermore, depending on the fiber size, different sorption capabilities were observed at varying pollutant viscosity. Large-sized fibers evidenced higher sorption performances on low viscosity pollutants. On the other hand, the best sorption capacity in high viscosity liquids were evidenced in small-sized AD fibers. Eventually, the adsorption properties were related to the microstructure and morphology of Arundo donax fibers scheming the main sorption mechanisms that acts during the pollutant uptake.

Although the sorption performances are lower than other conventional green materials, their oil/water selectivity makes them a cost-effective and reliable solution to oil spillage. Even if these results are promising, further focused studies in order to improve the knowledge on performances-morphology relationship and on surface treatments are welcome and at the same time improving the kinetic oil sorption capabilities and to reduce the water interaction of the vegetable fiber surface is another relevant issue for future activities.

Author Contributions: Conceptualization, L.C., E.P. and V.F.; methodology, L.C. and E.P.; validation, V.F.; formal analysis, L.C.; investigation, E.P.; data curation, L.C.; writing-original draft preparation, L.C., E.P. and V.F. writing-review and editing, L.C., E.P. and V.F.; supervision, V.F.

Funding: This research received no external funding.

Conflicts of Interest: The authors declare no conflict of interest.

\section{References}

1. Ariharasudhan, S.; Dhurai, B. Adsorption of oil from water surfaces using fibrous material-An overview. Man Made Text. India 2019, 47, 124-126.

2. Sun, R.C. Cereal Straw as a Resource for Sustainable Biomaterials and Biofuels, 1st ed.; Elsevier: Amsterdam, The Netherlands, 2010; ISBN 9780444532343.

3. Ceylan, D.; Dogu, S.; Karacik, B.; Yakan, S.D.; Okay, O.S.; Okay, O.; Karacık, B. Evaluation of Butyl Rubber as Sorbent Material for the Removal of Oil and Polycyclic Aromatic Hydrocarbons from Seawater. Environ. Sci. Technol. 2009, 43, 3846-3852. [CrossRef] [PubMed]

4. Wei, Q.; Mather, R.; Fotheringham, A.; Yang, R. Evaluation of nonwoven polypropylene oil sorbents in marine oil-spill recovery. Mar. Pollut. Bull. 2003, 46, 780-783. [CrossRef]

5. Nikkhah, A.A.; Zilouei, H.; Asadinezhad, A.; Keshavarz, A. Removal of oil from water using polyurethane foam modified with nanoclay. Chem. Eng. J. 2015, 262, 278-285. [CrossRef]

6. Feng, Y.; Xiao, C.F. Research on butyl methacrylate-lauryl methacrylate copolymeric fibers for oil absorbency. J. Appl. Polym. Sci. 2006, 101, 1248-1251. [CrossRef]

7. Wang, Z.; Lu, C.; Zhao, S.; Yang, L.; Li, X.; Yang, L. Hydrophobic Modification of Platanus Fruit Fibers as Natural Hollow Fibrous Sorbents for Oil Spill Cleanup. Water Air Soil Pollut. 2016, 227, 346.

8. Annunciado, T.; Sydenstricker, T.; Amico, S. Experimental investigation of various vegetable fibers as sorbent materials for oil spills. Mar. Pollut. Bull. 2005, 50, 1340-1346. [CrossRef] [PubMed] 
9. Adhithya, N.; Goel, M.; Das, A. Use of bamboo fiber in oil water separation. Int. J. Civ. Eng. Technol. 2017, 8, 925-931.

10. Liu, J.; Wang, X. A New Method to Prepare Oil Adsorbent Utilizing Waste Paper and Its Application for Oil Spill Clean-ups. BioResources 2019, 14, 3886-3898.

11. Zhong, L.; Tan, J.; Jing, S.; Cao, X.; Liu, C.F.; Wang, S.; Peng, X.; Chen, W.; Sun, R. An ultralight, elastic, cost-effective, and highly recyclable superabsorbent from microfibrillated cellulose fibers for oil spillage cleanup. J. Mater. Chem. A 2015, 3, 8772-8781.

12. Liu, Y.; Peng, Y.; Zhang, T.; Qiu, F.; Yuan, D. Superhydrophobic, ultralight and flexible biomass carbon aerogels derived from sisal fibers for highly efficient oil-water separation. Cellulose 2018, 25, 3067-3078. [CrossRef]

13. Feng, Y.; Liu, S.; Liu, G.; Yao, J. Facile and fast removal of oil through porous carbon spheres derived from the fruit of Liquidambar formosana. Chemosphere 2017, 170, 68-74. [CrossRef]

14. Husseien, M.; Amer, A.A.; El-Maghraby, A.; Taha, N.A. Availability of barley straw application on oil spill clean up. Int. J. Environ. Sci. Technol. 2009, 6, 123-130. [CrossRef]

15. Dong, T.; Xu, G.; Wang, F. Adsorption and adhesiveness of kapok fiber to different oils. J. Hazard. Mater. 2015, 296, 101-111. [CrossRef]

16. Said, A.; Ludwick, A.; Aglan, H. Usefulness of raw bagasse for oil absorption: A comparison of raw and acylated bagasse and their components. Bioresour. Technol. 2009, 100, 2219-2222. [CrossRef] [PubMed]

17. Perdue, R.E. Arundo donax — Source of musical reeds and industrial cellulose. Econ. Bot. 1958, 12, 368-404. [CrossRef]

18. Sharma, K.; Kushwaha, S.P.S.; Gopal, B. A comparative study of stand structure and standing crops of two wetland species, Arundo donax and Phragmites karka, and primary production in Arundo donax with observations on the effect of clipping. Trop. Ecol. 1998, 39, 3-14.

19. Weidenfeller, B.; Lambri, O.A.; Bonifacich, F.G.; Arlic, U.; Gargicevich, D. Damping of the Woodwind Instrument Reed Material Arundo donax L. Mater. Res. 2018, 21. [CrossRef]

20. Obataya, E.; Umezawa, T.; Nakatsubo, F.; Norimoto, M. The Effects of Water Soluble Extractives on the Acoustic Properties of Reed (Arundo donax L.). Holzforschung 1999, 53, 63-67. [CrossRef]

21. Pilu, R. Giant reed (Arundo donax L.): A weed plant or a promising energy crop? Afr. J. Biotechnol. 2012, 11,9163-9174.

22. Ververis, C.; Georghiou, K.; Christodoulakis, N.; Santas, P.; Santas, R. Fiber dimensions, lignin and cellulose content of various plant materials and their suitability for paper production. Ind. Crop. Prod. 2004, 19, 245-254. [CrossRef]

23. Papazoglou, E.; Karantounias, G.; Vemmos, S.; Bouranis, D. Photosynthesis and growth responses of giant reed (Arundo donax L.) to the heavy metals Cd and Ni. Environ. Int. 2005, 31, 243-249. [CrossRef]

24. Flores, J.; Pastor, J.; Martinez-Gabarron, A.; Gimeno-Blanes, F.; Rodríguez-Guisado, I.; Frutos, M. Arundo donax chipboard based on urea-formaldehyde resin using under $4 \mathrm{~mm}$ particles size meets the standard criteria for indoor use. Ind. Crop. Prod. 2011, 34, 1538-1542. [CrossRef]

25. Fiore, V.; Scalici, T.; Valenza, A. Characterization of a new natural fiber from Arundo donax L. as potential reinforcement of polymer composites. Carbohydr. Polym. 2014, 106, 77-83. [CrossRef] [PubMed]

26. Fiore, V.; Scalici, T.; Vitale, G.; Valenza, A. Static and dynamic mechanical properties of Arundo donax fillers-epoxy composites. Mater. Des. 2014, 57, 456-464. [CrossRef]

27. Fiore, V.; Botta, L.; Scaffaro, R.; Valenza, A.; Pirrotta, A. PLA based biocomposites reinforced with Arundo donax fillers. Compos. Sci. Technol. 2014, 105, 110-117. [CrossRef]

28. Scalici, T.; Fiore, V.; Valenza, A. Effect of plasma treatment on the properties of Arundo Donax L. leaf fibres and its bio-based epoxy composites: A preliminary study. Compos. Part B Eng. 2016, 94, 167-175. [CrossRef]

29. Ismail, Z.Z.; Jaeel, A.J. A novel use of undesirable wild giant reed biomass to replace aggregate in concrete. Constr. Build. Mater. 2014, 67,68-73. [CrossRef]

30. De Rosa, I.M.; Kenny, J.M.; Puglia, D.; Santulli, C.; Sarasini, F. Morphological, thermal and mechanical characterization of okra (Abelmoschus esculentus) fibres as potential reinforcement in polymer composites. Compos. Sci. Technol. 2010, 70, 116-122. [CrossRef]

31. Cheu, S.C.; Kong, H.; Song, S.T.; Johari, K.; Saman, N.; Yunus, M.A.C.; Mat, H. Separation of dissolved oil from aqueous solution by sorption onto acetylated lignocellulosic biomass-Equilibrium, kinetics and mechanism studies. J. Environ. Chem. Eng. 2016, 4, 864-881. [CrossRef] 
32. Wahi, R.; Abdullah, L.C.; Mobarekeh, M.N.; Ngaini, Z.; Yaw, T.C.S. Utilization of esterified sago bark fibre waste for removal of oil from palm oil mill effluent. J. Environ. Chem. Eng. 2017, 5, 170-177. [CrossRef]

33. Aloulou, F.; Boufi, S.; Labidi, J. Modified cellulose fibres for adsorption of organic compound in aqueous solution. Sep. Purif. Technol. 2006, 52, 332-342. [CrossRef]

34. Nishi, Y.; Iwashita, N.; Sawada, Y.; Inagaki, M. Sorption kinetics of heavy oil into porous carbons. Water Res. 2002, 36, 5029-5036. [CrossRef]

35. El-Din, G.A.; Amer, A.; Malsh, G.; Hussein, M. Study on the use of banana peels for oil spill removal. Alex. Eng. J. 2018, 57, 2061-2068. [CrossRef]

36. Piperopoulos, E.; Calabrese, L.; Mastronardo, E.; Proverbio, E.; Milone, C. Synthesis of reusable silicone foam containing carbon nanotubes for oil spill remediation. J. Appl. Polym. Sci. 2018, 135, 46067. [CrossRef]

37. Dong, T.; Cao, S.; Xu, G. Highly efficient and recyclable depth filtrating system using structured kapok filters for oil removal and recovery from wastewater. J. Hazard. Mater. 2017, 321, 859-867. [CrossRef]

38. Piperopoulos, E.; Calabrese, L.; Mastronardo, E.; Abdul Rahim, S.H.; Proverbio, E.; Milone, C. Assessment of sorption kinetics of carbon nanotube-based composite foams for oil recovery application. J. Appl. Polym. Sci. 2019, 136, 47374. [CrossRef]

39. Wang, N.; Deng, Z. Synthesis of magnetic, durable and superhydrophobic carbon sponges for oil/water separation. Mater. Res. Bull. 2019, 115, 19-26. [CrossRef]

40. Abdelwahab, O.; Nasr, S.M.; Thabet, W.M. Palm fibers and modified palm fibers adsorbents for different oils. Alex. Eng. J. 2017, 56, 749-755. [CrossRef]

41. Baker, R.W. Membrane Technology and Applications; Wiley: Chichester, UK, 2004.

42. Cui, Y.; Xu, G.; Liu, Y. Oil sorption mechanism and capability of cattail fiber assembly. J. Ind. Text. 2014, 43, 330-337. [CrossRef]

(C) 2019 by the authors. Licensee MDPI, Basel, Switzerland. This article is an open access article distributed under the terms and conditions of the Creative Commons Attribution (CC BY) license (http://creativecommons.org/licenses/by/4.0/). 